\title{
Empirical Studies of Pen Tilting Performance in Pen-based User Interfaces
}

\author{
Feng Tian \\ Fei Lu \\ Intelligence Engineering Lab, Institute Intelligence Engineering Lab, Institute \\ of Software, Chinese Academy of of Software, Chinese Academy of \\ Sciences, China \\ tf@iel.iscas.ac.cn \\ Xiang Cao \\ Sciences, China \\ Ivfei@iel.iscas.ac.cn \\ Guozhong Dai \\ Microsoft Research Asia, China \\ State Key Lab. of Computer Science, \\ Institute of Software, Chinese \\ Academy of Sciences, China \\ dgz@iel.iscas.ac.cn \\ Xiaolong Zhang \\ Pennsylvania State University, \\ USA \\ Izhang@ist.psu.edu \\ Hongan Wang \\ xiangc@microsoft.com \\ Lab. of Computer Science, \\ Institute of Software, Chinese \\ Academy of Sciences, China \\ wha@iel.iscas.ac.cn
}

\begin{abstract}
Recently, pen tilting has been explored in pen-based user interfaces and has shown potential to improve user interaction in various tasks (e.g., menu selection, modeless object manipulation). However, some basic questions concerning pen tilting behaviors, such as the ideal range, azimuth size, and direction of pen tilting, have not been thoroughly investigated. In this paper, we report our empirical studies on user performances in basic pen tilting tasks. First, we conducted a baseline study, which helps us to determine tilting directions, tilting ranges, and the thresholds that separate incidental pen tilting actions from intentional actions used for interaction. Based on the results from the baseline study, we designed an experiment to investigate user performances in goal tilting in different tilting ranges, azimuth sizes, and directions. Drawing on the results of our data analyses on task completion time, error rate, and pen tip movements, we discussed values of tilting parameters like titling range, minimal azimuth size, and tilting direction.
\end{abstract}

\section{Categories and Subject Descriptors}

H.5.2 [Information Interfaces and Presentation]: User Interfaces-Graphical user interfaces, input devices and strategies, interaction styles, theory and methods; H.1.2 [Models and Principles]: User/Machine Systems-Human factors, human information processing; I.3.6 [Computer Graphics]: Methodology and Techniques-Interaction technique

\section{General Terms}

Empirical evaluation, pen-based user interface, pen tilting.

\section{Keywords}

Empirical evaluation, pen-based user interface, pen tilting

Permission to make digital or hard copies of all or part of this work for personal or classroom use is granted without fee provided that copies are not made or distributed for profit or commercial advantage and that copies bear this notice and the full citation on the first page. To copy otherwise, or republish, to post on servers or to redistribute to lists, requires prior specific permission and/or a fee.

VINCI 2011, August 4-5, 2011, Hong Kong, China.

Copyright @ 2011 ACM 978-1-4503-0875-5/11/08...\$10.00.

\section{INTRODUCTION}

Pen-based interactions have gained strong attention for its naturalness and intuitiveness in recent years. Currently, the input dimensions we may get from a digital pen go beyond the traditional 2D position of a pen tip, and also include advanced information such as pen hover, pen-tip pressure, pen 3D orientation, and pen rotation. Many research projects have explored various approaches to incorporate advanced pen information seamlessly with the 2D position of the pen tip to enrich pen-based interaction [2][8][14][18].

Pen tilting is one of such efforts. Pen titling uses the 3D orientation of a pen, which consists of the altitude angle and the azimuth angle, as shown in Figure 1. Designs based on pen tilting include Tilt Cursor [18], which visualizes the pen orientation to improve the Stimulus-Response Compatibility of a digital pen, Tilt Menu [19], which facilitates menu selection with pen tilting behaviors, and a system to integrate pen shaking gestures to support user interaction [17]. These projects have shown that pen tilting techniques have the potential to aid traditional one-handed techniques by allowing two simultaneous inputs (e.g., executing a command and selecting an associated parameter) without involving the second hand or another device. Designs based on pen tilting can offer benefits to many user activities, such as modeless control, drag-n-drop, copy-n-paste, and multi-parameter inputting.

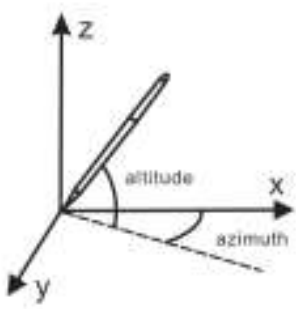

Figure 1: 3D orientation information of pen.

Despite these innovative designs, some questions concerning basic user behaviors in pen tilting have not been thoroughly investigated. Some efforts had been made [19][24], but these projects focused on high-level user tasks (e.g., menu selection) and offered little insight into the relationship between tilting 
factors (e.g., titling range and direction) and human performances (e.g., tilting speed and accuracy). A good understanding of this relationship can inform the design of pen tilting tools and help to generalize the design in broader application domains.

Inspired by the Fitts' Law study (Fitts, 1954), we examined user behaviors through a basic task that is involved in almost all tiltingbased design - goal tilting. We studied user performances in different conditions, such as tilting ranges, target azimuth sizes, and tilting directions, and identified some baseline parameters that pen-tilt-based designs can follow.

This paper is structured as follows. First, we review relevant literature. Then, we report a baseline study that provides us with important parameters that will be used in our main experiment on user performances in target reaching with pen tilting. Next, we describe the design of the experiment and the data analyses on the relations among task completion time, the tilting range, and the azimuth size. After discussing our results, the paper concludes with future research directions.

\section{RELATED WORK}

The tilt information of a pen can be applied in many fields to improve user interaction. The Rockin' Mouse, a device for both 2D and 3D interaction, used tilt input to integrate the 3D movement of an input device in 2D object manipulation [2]. Rekimoto [16] focused on the use of tilt information of small screen computers to build interaction techniques like pull-down menus, scroll bars, map browsing and 3D object viewing. Some other work, such as TiltType [13], TiltText [21], used tilt information of a phone to do text entry for mobile devices.

In pen-based user interface design, the Tilt Cursor [18] is a type of cursor that dynamically reshapes itself to provide the 3D orientation cue of a pen and provides better stimulus-response compatibility in stylus-based and tablet devices. The Tilt Menu [19] supports menu selection with the pen's 3D orientation information and without involving any pen tip movements. As a one-handed technique, the Tilt Menu can merge command selection and direct manipulation in freeform drawing tasks.

The recent work by Rahman et al. [15] analyzes the design space of wrist-based interactions with a focus on the levels of control possibility. Results show that users can control comfortably at least 16 levels on the pronation/supination axis and that using a quadratic mapping function for discretization of tilt space significantly improves user performance across all tilt axes. This research also indicates that input techniques such as the Tilt Cursor and Tilt Menu, employ different motor control skills from techniques based on force grip tilting, like holding a cell phone. Thus, different design principles are required for these two kinds of tilting.

Directly applying these existing theories and models in pen-tailbased designs may be inappropriate. This is because pen tilting involves different motor control skills from those traditional pointing or steering techniques do [22][4]. Research is needed to investigate the characteristics of pen tail tilting.

\section{RESEARCH OVERVIEW}

Our research included work in two phases. In Phase I, we conducted a baseline study to obtain some basic parameters that are critical to the understanding and identification of pen-tilting activities. First, it is important to differentiate intentional tilting actions from incidentally tilting while users perform other tasks with the pen tip. By identifying the tilting threshold that distinguishes intentional tilting from incidental tilting, we can detect tilting activities more accurately in the experiment. Second, it is needed to understand people's natural pen-hold positions. The distribution of natural pen-holding postures in writing and tracing tasks can help to set up the tilting directions in our experiment to focus on the effects of on-axis and off-axis directions [9].

Based on the research results from Phase I, we studied user performances in goal tilting in Phase II. In this phase, we conducted experiments to measured user performances, in terms of task completion time, error rate and pen tip movements in goal tilting under different pen tilting parameters, including tilting ranges, target azimuth sizes, and tilting directions. Based on the collected data, we explored the relationship among the task completion time, tilting distance, and the size of goal.

\section{PHASE I: BASELINE STUDY ON INCIDENTAL TILTING AND PEN- HOLDING POSTURE}

This study had two goals: 1) to identify the threshold that distinguishes intentional tilting from incidental tilting, and 2) to determine the natural pen-holding postures during typical pen operations such as tracing (e.g., navigating a menu) and writing. A straight line tracing task was chosen to represent trajectory-based interactions such as dragging or menu navigation.

\section{Experimental Design}

Two types of tasks - tracing a line and writing down a given sentence-were used in the study. In a tracing task, participants needed to trace a line from the start to the end using the pen tip. This task represents typical pen interaction tasks in drawing. Figure 2 illustrates a tracing task. The starting location of a line is shown as a red circle, and the end is a green circle. A black arrow is used to represent the tilt cursor, and its head and tail correspond to the $2 \mathrm{D}$ projections of the pen tip and tail.

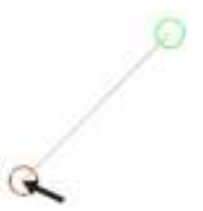

(a)

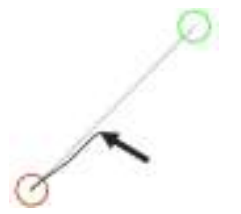

(b)

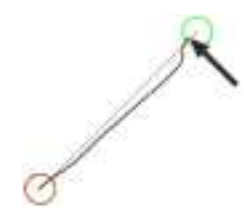

(c)
Figure 2: Line tracing task. (a) task initiated; (b) task in progress; (c) task completed.

Lines used in the study varied in length and direction. Three line lengths were offered: 15 pixels, 25 pixels, and 50 pixels. Lines were positioned in eight directions: north $(\mathrm{N})$, east (E), south (S), west (W), north-east (NE), north-west (NW), south-east (SE), and south-west (SW). All these directions were defined with respect to the screen. Figure 3 shows the eight directions.

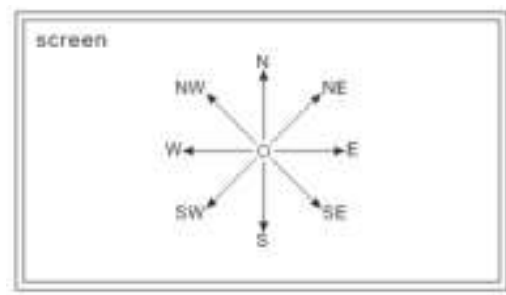

Figure 3: Eight tilting directions in line tracing. 
In a writing task, participants were asked to transcribe sentences displayed on the top of the screen with the pen tip. This task represents typical pen interaction tasks such as handwriting text input or note taking. Sentences were in two languages - English and Chinese, and were presented in random order.

Twelve subjects were recruited for the study. All of them were right-handed. Each participant was asked to do tracing tasks (3 line lengths $\mathrm{x} 8$ directions $\mathrm{x} 3$ trials) and writing tasks (8 trials) at their natural speeds.

For each task trial, data on pen tilting range, tilting speed, altitude angle, and azimuth angle were collected. Tilting range is defined as the difference between the maximum and minimum altitude angle during a task. Tilting speed refers to the velocity of pen tail in the change of the altitude angle. The altitude angle and azimuth angle of a pen in line tracing and writing will be recorded to help determine the natural pen-holding posture.

The study was conducted on a 19" LCD screen with the resolution of $1024 \times 768$ pixels and a Wacom Intuos $36 " \times 11$ " digitizing tablet with a stylus pen.

\section{Results}

\section{Incidental \& Intentional Tilting}

Experiment results allowed us to characterize incidental tilting by tilting ranges and speeds. Data show that $92.5 \%$ of pen movement actions had a tilting speed smaller than $35 \%$ s, and that $97.7 \%$ of actions had a tilting range smaller than $20^{\circ}$. If both variables are considered, $99.9 \%$ of pen movement had a tilting speed below $35 \%$ or a tilting range below $20^{\circ}$. Relatively small values of tilting speed and tilting range suggest that users do not tilt a pen dramatically when performing regular tasks. We can identify tilting actions with either a tilting speed smaller than $35 \%$ or a tilting range smaller than $20^{\circ}$ as incidental tilting. Conversely, tilting actions with a speed faster than $35^{\circ}$ s and a range above $20^{\circ}$ can therefore be classified as intentional tilting. Therefore, we define the minimal intentional tilting range as $20^{\circ}$. This value may guide us for future design of tilting techniques.

\subsubsection{Pen-holding Posture}

Knowing the natural pen-holding posture of a user is important to analyze pen tilting behaviors, such as identifying the azimuth angle regions that are easy or difficult to reach. A natural penholding posture can be determined by the altitude angle and the azimuth angle. Our data showed that the average of the altitude angle is $53.83^{\circ}\left(\mathrm{SD}=5.50^{\circ}\right)$ and the average azimuth is $45.43^{\circ}$ $\left(\mathrm{SD}=20.95^{\circ}\right)$. This result suggests that if tilting directions defined in Figure 3 can be rotated by $45^{\circ}$ counter clockwise, the natural pen-holding posture will be aligned with the north-south tilting direction. The new tilting directions after rotation are shown in Figure 4.

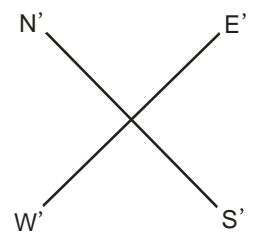

Figure 4. Adjusted tilting directions.

\subsubsection{Maximum Easily Tilting Range}

We defined the Maximum Easily Tilting Range as $35^{\circ}$. The reason is based on an obvious fact that when users holding pen in a static posture, they hardly continue tilt their pen along the direction of the current azimuth value. It means the maximum tilting range should lower that $90^{\circ}$ subtract currently altitude angle of PenHolding Posture $\left(90^{\circ}-53.83^{\circ}\right)$. In order to simplify the design of following experiments, we choose $35^{\circ}$ as the value which is very close to the actual value $36.17^{\circ}$.

\section{PHASE 2: GOAL TILTING EXPERIMENT}

To evaluate the usability performance of the tilting actions, we conducted a pen-tilting experiment. The goal of the experiment was to examine the following five issues:

(1) The impact of tilting ranges on tilting task performance;

(2) The impact of target azimuth sizes on titling task performance;

(3) The impact of tilting directions on tilting task performance;

(4) The relationships between pen tilting and unintentional pen tip movement;

(5) The relationship among task competition time, the distance to the object, and the width of object, if there is any.

\section{Participants and Apparatus}

Twelve people (eight female and four male) participated in the experiment. Participants were all right-handed and familiar with computers. All of them had prior experience using pen and tablet. The experiment was run on a 19" LCD screen with the resolution of $1024 \times 768$ pixels and a Wacom Intuos3 $6 " \times 11$ " digitizing tablet with a stylus pen. A Tilt Cursor [18] was used to provide feedback about the position, altitude, and azimuth angle of the pen.

\section{Task}

The task of the experiment was to tilt the pen tail to reach a goal. Figure 5 shows a goal tilting task. For each task, a participant saw a starting point, which is specified by two crossed lines and a circle, and a goal arc. The participant needed to first position the pen tip inside the circle surrounding the starting point and then tilt the pen tail to reach the arc. A tilt cursor was used to indicate the tilting process.

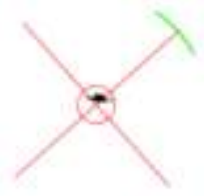

(a) task initiated

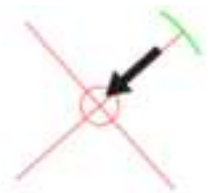

(b) task in progress

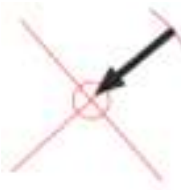

(c) task completed
Figure 5. Goal crossing task. The black arrow is the tilt cursor.

\section{Design}

The experiment was a within-subjects, factorial design with repeated measures. Three factors were involved: tilting direction, arc size, and tilting range. Four tilting directions (N', S', E' and W') were used. The goal arcs had four different azimuth sizes: $30^{\circ}, 45^{\circ}, 60^{\circ}$, and $90^{\circ}$ ). Five tilting ranges were applied: $15^{\circ}, 20^{\circ}$, $25^{\circ}, 30^{\circ}$, and $35^{\circ}$. For each tilting direction, participants performed five blocks of 40 tasks. Each task crossed an arc size and a tilting range and appeared twice in each block. These tasks were randomized. Four tilting directions were counterbalanced. 


\section{Procedure}

When a task was presented on the screen, a participant saw a starting point and a goal arc. The participant needed first to place the pen tip on the Wacom tablet and point it to the cross of the starting point, and then to tilt the pen to a vertical position so that the Tilt Cursor was within the circle surrounding the starting point. The radius of the circle was determined in such a way that the vertical position error of the pen was less than $2^{\circ}$. As soon as the tilt cursor entered the circle, the color of the circle changed, indicating that the task had begun and the participant needed to tilt the pen towards the goal arc. As soon as the Tilt Cursor reached the goal, the arc turned to red from green, indicating the end of the task.

Subjects were told to complete the trials as quickly and accurately as possible. Participants could have a break between blocks. The experiment lasted 1 hour for each participant.

For each task, we collected data on task completion time, error rate, and pen tip movement. Task completion time is the time between the moment the tilt cursor touched the circle and the moment the Tilt Cursor reached a goal. An error was recorded when the pen tip was lifted from the Wacom pad before a task was finished or tilting was out of the altitude range of the target arc. Error rate is the percentage of trials for a particular condition that resulted error. For pen tip movement, we recorded the distance the pen tip had traveled from the moment it touched the screen to the time it was lifted. The length of the whole tip trajectory during the task was calculated as the pen tip movement.

\section{Results}

All participants completed their tasks. In total, we collected data of 800 trails from each participant.

\section{Task Completion Time}

\section{Main Effects of Factors}

The means of task completion time of four directions (E', S', W', $\left.\mathrm{N}^{\prime}\right)$ are $605.27 \mathrm{~ms}, 751.21 \mathrm{~ms}, 624.84 \mathrm{~ms}$, and $632.83 \mathrm{~ms}$ respectively (Figure 6). A repeated measures analysis of variance showed a significant main effect of tilting direction (F3, $33=$ 15.96, $\mathrm{p}<.001)$. Pairwise comparisons showed that the completion time of the direction $\mathrm{S}^{\prime}$ is significantly longer than that of other directions ( $\mathrm{p}<=.001$ ), while non-significant differences were found between E', N' and W' ( $p>=.05)$.

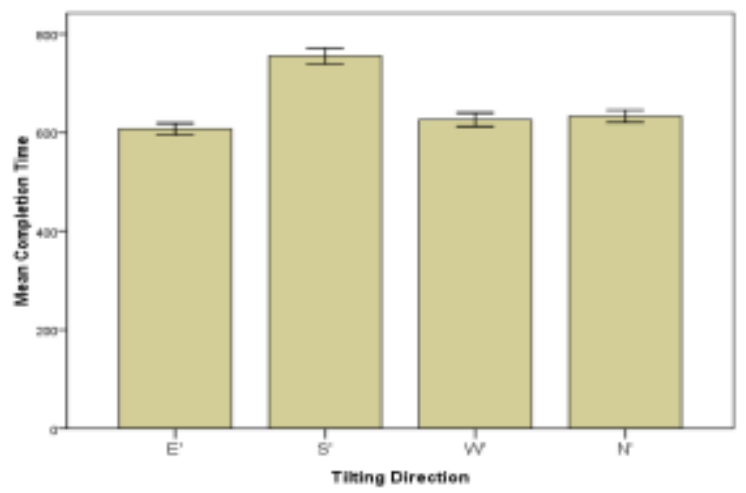

Figure 6: Means of task completion time for 4 tilting directions.

The means of task completion time for five tilting ranges $\left(15^{\circ}\right.$, $20^{\circ}, 25^{\circ}, 30^{\circ}, 3^{\circ}$ ) are $530.80 \mathrm{~ms}, 573.12 \mathrm{~ms}, 634.77 \mathrm{~ms}, 713.07 \mathrm{~ms}$, 815.94ms respectively (Figure 7). Repeated measures ANOVA showed a significant main effect of tilting range (F4, $44=67.96$, $\mathrm{p}<.001)$. Pairwise comparisons showed that the completion time of each larger tilting range is significantly longer than that of other smaller tilting ranges $(\mathrm{p}<.01)$.

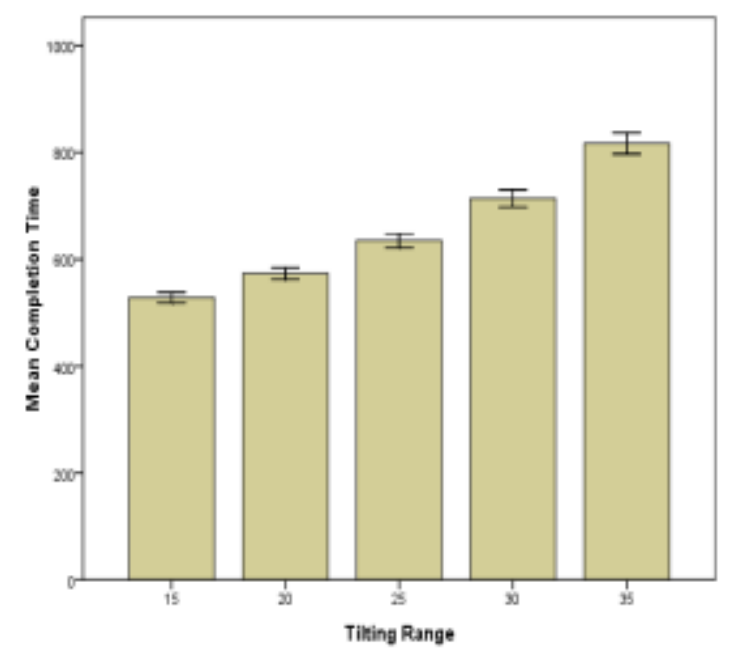

Figure 7. Means of task completion time in different tilting ranges.

The means of task completion time of four azimuth size values $\left(30^{\circ}, 45^{\circ}, 60^{\circ}, 90^{\circ}\right)$ are $705.45,670.72,643.41$, and 594.57 respectively (Figure 8). Repeated measures ANOVA indicates a significant main effect of azimuth on task completion time (F3, 33 $=14.99, \mathrm{p}<.001)$. Pairwise comparisons showed that the completion time of each larger target azimuth size is significantly shorter than that of other smaller target azimuth sizes $(\mathrm{p}<.05)$.

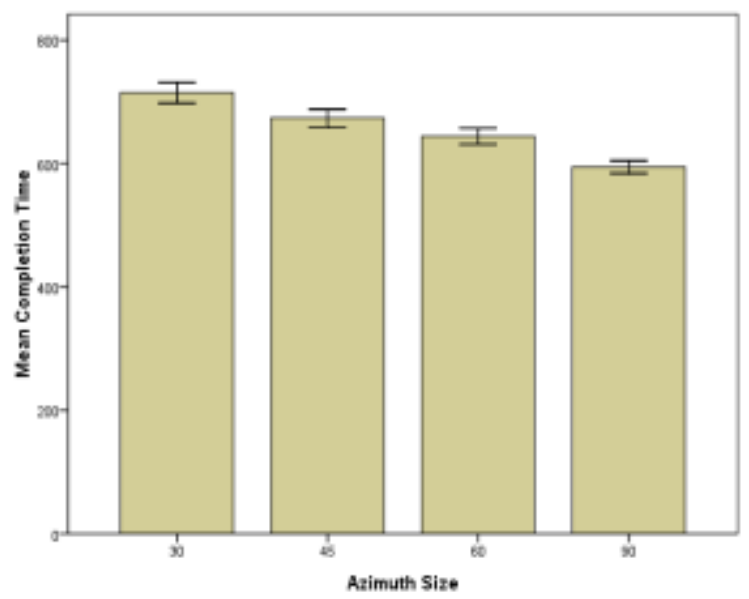

Figure 8. Means of task completion time on different target azimuth sizes

\section{Interaction among Factors}

Repeated measures ANOVA showed interaction effects between tilting range and tilting direction $(\mathrm{p}<.0001)$ (Figure 9), between target azimuth size and tilting direction $(\mathrm{p}<$.0001) (Figure 10), and between tilting range and target azimuth size $(\mathrm{p}<.0001)$ (Figure 11). 


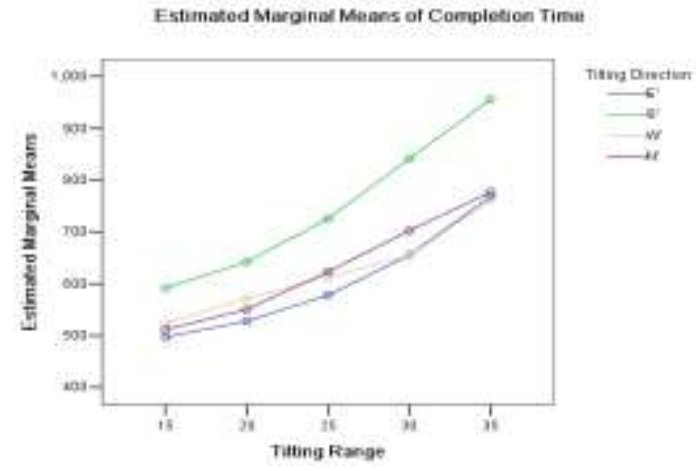

Figure 9. Task completion time comparison (Tilting Direction vs. Tilting Range)

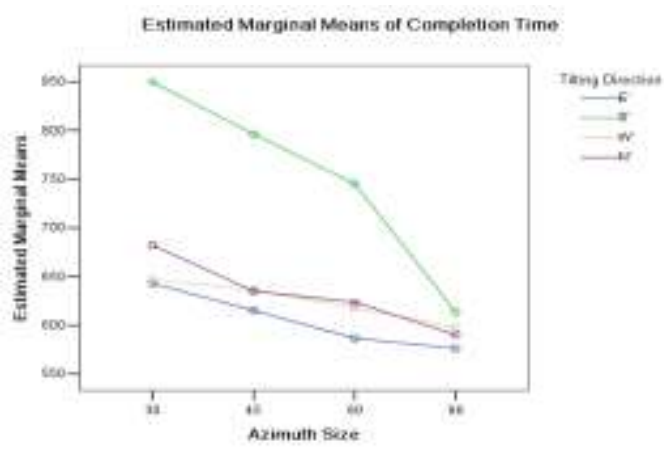

Figure 10. Task completion time comparison (Azimuth Size vs. Tilting Direction).

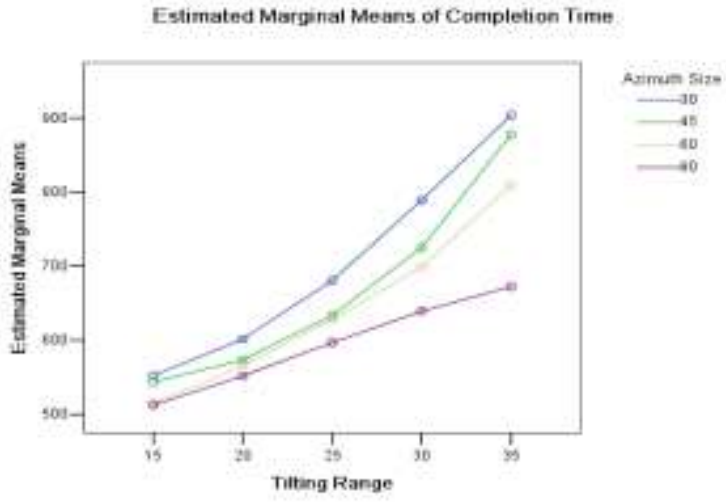

Figure 11. Task completion time comparison (Azimuth Size vs. Tilting Range).

Considering the interaction effects above, we conducted further analysis of simple effects of these factors. It will provide us the detail guides on designing pen-based tilting techniques.

First, we analyzed the completion time of four directions (E', S', W', N') in five tilting ranges $\left(15^{\circ}, 20^{\circ}, 25^{\circ}, 30^{\circ}, 35^{\circ}\right)$ and four azimuth sizes $\left(30^{\circ}, 45^{\circ}, 60^{\circ}, 90^{\circ}\right)$. We found significant effects of direction in all tilting ranges $(\mathrm{p}<.0001)$; besides, significant effects were found $(p<.0001)$ in different azimuth sizes, except in azimuth size $90^{\circ}(\mathrm{p}=.186)$. These results are similar to our previous research on Tilt Menu, in which non-significant differences between slices were found when the Tilt Menu has four slices (each slice is $90^{\circ}$ ).

Then we analyzed the mean completion times of five tilting ranges in different azimuth sizes and tilting directions. Significant main effects of tilting range were found in all azimuth sizes $\left(30^{\circ}\right.$, $\left.45^{\circ}, 60^{\circ}, 90^{\circ}\right)(\mathrm{p}<.0001)$, as well as in all tilting directions( $\mathrm{E}^{\prime}, \mathrm{S}$ ' $\left.\mathrm{W}^{\prime}, \mathrm{N}^{\prime}\right)(\mathrm{p}<.0001)$.

We also analyzed the mean completion times of target azimuth size in four directions(E', S', W' and N') and five tilting range $\left(15^{\circ}, 20^{\circ}, 25^{\circ}, 30^{\circ}, 35^{\circ}\right)$. Significant differences of target azimuth size were found in all tilting directions $(\mathrm{p}<.0001)$. As for tilting range, the mean completion times of target azimuth in tilting range $25^{\circ}, 30^{\circ}$ and $35^{\circ}$ were significant different $(\mathrm{p}<.05)$, while that in tilting range $15^{\circ}$ and $20^{\circ}$ were not significant different $(\mathrm{p}=.132, \mathrm{p}=.066)$.

\section{Error Rate}

\section{Main Effects of Factors}

The means of error rate for four directions (E', S', W', N') are $5.33 \%, 6.97 \%, 5.67 \%$, and $6.92 \%$, respectively (Figure 12). Repeated measures ANOVA showed non-significant main effect of direction on error rate $(F 3,33=2.57, p=.071)$, and due to all error rates are lower than $10 \%$, we do not do pairwise comparisons further.

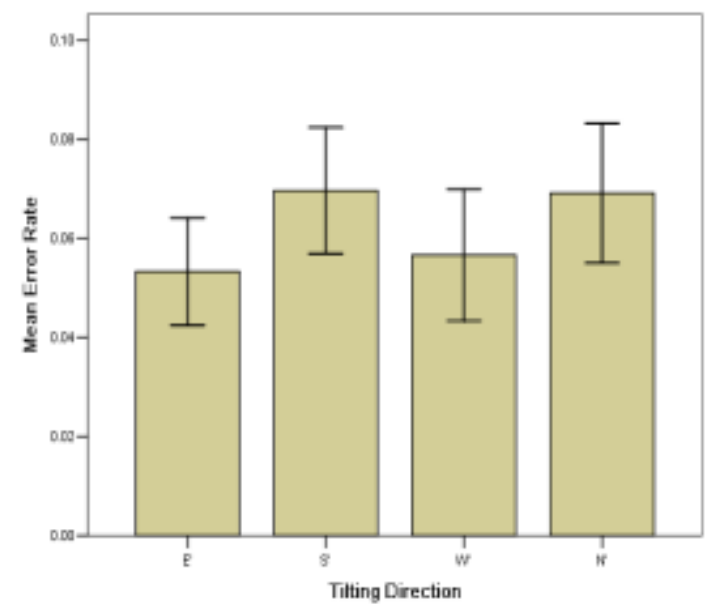

Figure 12: Mean error rates for 4 directions with $95 \%$ confidence intervals.

The mean error rates for five tilting ranges $\left(15^{\circ}, 20^{\circ}, 25^{\circ}, 30^{\circ}\right.$, $35^{\circ}$ ) are $10.10 \%, 6.20 \%, 5.26 \%, 4.74 \%$, and $4.79 \%$ respectively (Figure 13). Repeated measures ANOVA showed a significant main effect of tilting range on error rate $(F 4,44=12.35$, $p<$ .0001). Pairwise comparisons reveal that the error rate of $15^{\circ}$ is significantly higher than that of others $(p<.05)$, while nonsignificant differences were found between others. 


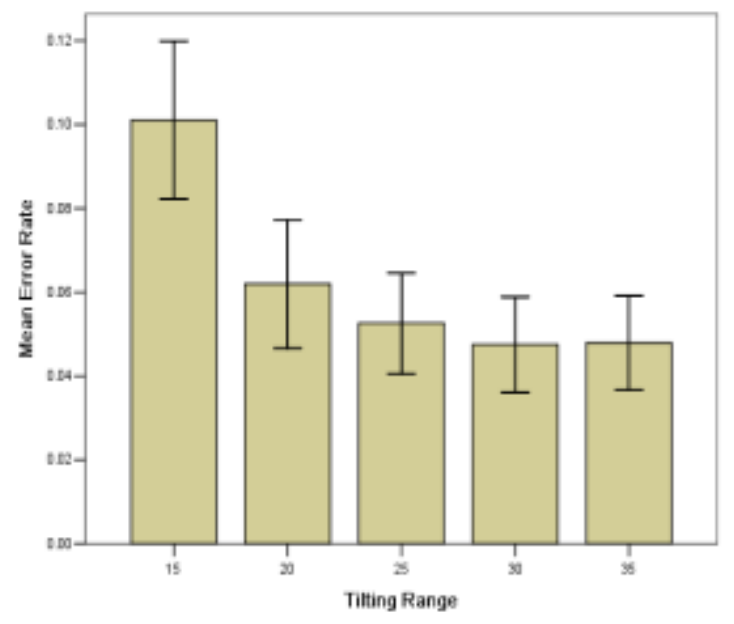

Figure 13. Mean error rates on different tilting ranges with $95 \%$ confidence intervals.

The average error rates for four azimuth size values $\left(30^{\circ}, 45^{\circ}, 60^{\circ}\right.$, $90^{\circ}$ ) are $12.46 \%, 6.21 \%, 3.29 \%$, and $2.92 \%$ respectively (Figure 14). Repeated measures ANOVA showed a significant main effect of azimuth on error rate (F3, $33=32.32$, $\mathrm{p}<.0001)$. Pairwise comparisons indicates that the error rate of $30^{\circ}$ is significantly higher than that of others $(\mathrm{p}<.0001)$, and the error rate of $45^{\circ}$ is significantly higher than that of $60^{\circ}$ and $90^{\circ}(\mathrm{p}<.0001)$. Nonsignificant difference was found between $60^{\circ}$ and $90^{\circ}(\mathrm{p}=.495)$.

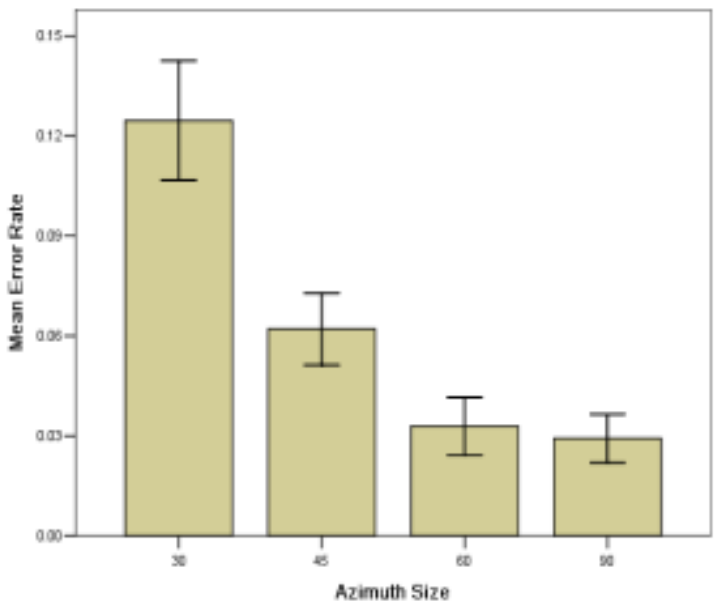

Figure 14. Mean error rates on different target azimuth sizes with $95 \%$ confidence intervals.

\section{Interaction among Factors}

Repeated measures ANOVA showed no interaction effects between tilting range and directions $(p=.955)$, while significant effects are found in target azimuth sizes vs. directions ( $\mathrm{p}=.037$ ), and tilting ranges vs. target azimuth sizes $(\mathrm{p}<.0001)$, as shown in Figure 15, 16 and 17 respectively.

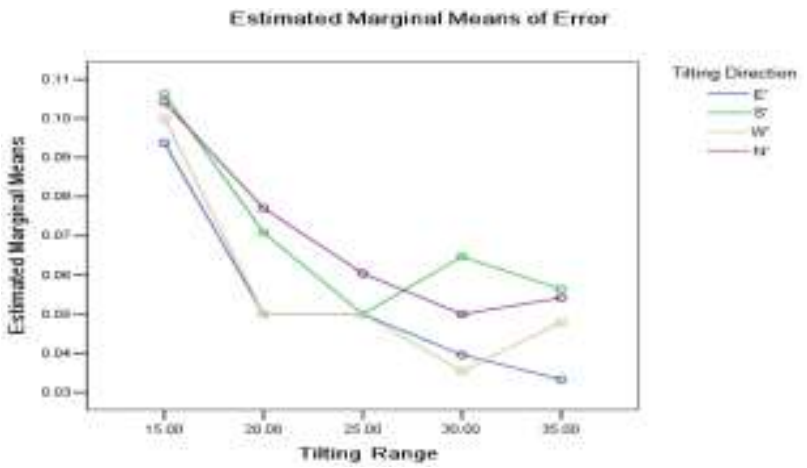

Figure 15. Interaction effects between tilting ranges and directions in error rate.

Espmated Margieat means of Frro

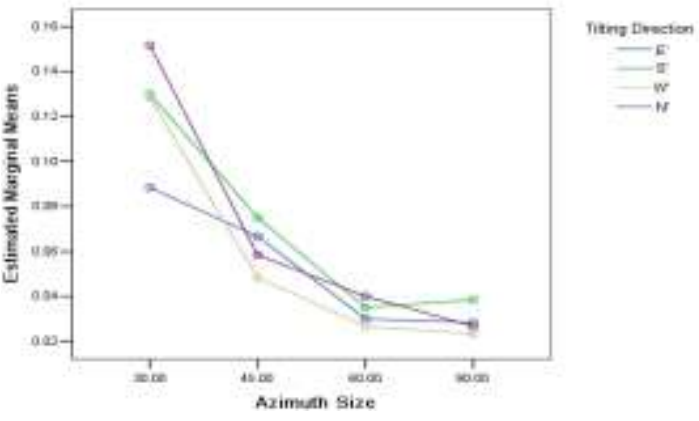

Figure 16. Interaction effects between target azimuth sizes and directions in error rate.

Estimated Marginal Means of Erros

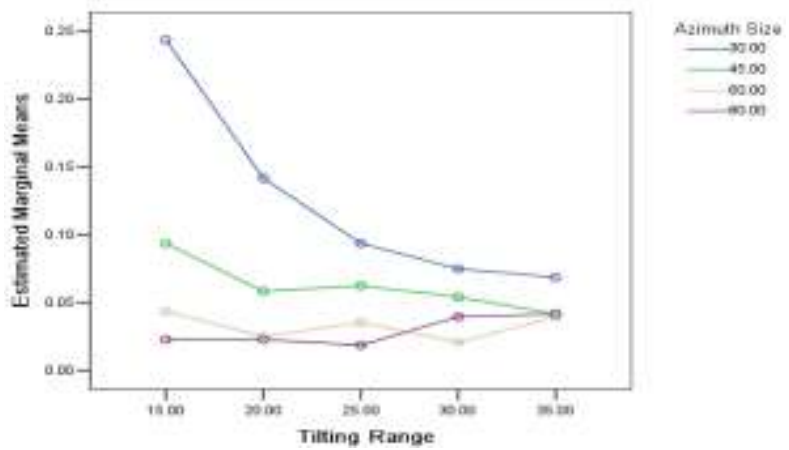

Figure 17. Interaction effects between tilting ranges and target azimuth sizes in error rate.

Considering the interaction effects between target azimuth size and tilting direction, and between tilting range and target azimuth size, we analyzed simple effects of these factors. It will provide us the detail guides on designing pen-based tilting techniques.

First, we analyzed the mean error rates of four tilting directions $\left(E^{\prime}, S^{\prime}, W^{\prime}, N^{\prime}\right)$ in four target azimuth sizes $\left(30^{\circ}, 45^{\circ}, 60^{\circ}, 90^{\circ}\right)$. In target azimuth size $30^{\circ}$, there was significant main effect of tilting direction $(p=.001)$. The error rate of the direction $E^{\prime}$ is significantly lower than that of other directions $(\mathrm{p}<=.005)$, while non-significant differences were found between N', W' and S'. When the target azimuth size value are above $30^{\circ}\left(45^{\circ}, 60^{\circ}, 90^{\circ}\right)$, there were no significant main effects $(\mathrm{p}=.395, \mathrm{p}=.854, \mathrm{p}=$ $.800)$

We also analyzed the mean error rates of five tilting ranges $\left(15^{\circ}\right.$, $\left.20^{\circ}, 25^{\circ}, 20^{\circ}, 35^{\circ}\right)$ in four target azimuth sizes $\left(30^{\circ}, 45^{\circ}, 60^{\circ}\right.$, $90^{\circ}$ ). In target azimuth size $30^{\circ}$, it showed significant main effect 
of tilting range $(\mathrm{p}<.0001)$; in contrast, when the azimuth size is above $30^{\circ}\left(45^{\circ}, 60^{\circ}, 90^{\circ}\right)$, it showed no significant main effects of tilting range $(\mathrm{p}=.059, \mathrm{p}=.681, \mathrm{p}=.389)$.

Finally, we analyzed the mean error rates of four azimuth sizes $\left(30^{\circ}, 45^{\circ}, 60^{\circ}, 90^{\circ}\right)$ in four tilting directions(E', S', W', N') and five tilting ranges $\left(15^{\circ}, 20^{\circ}, 25^{\circ}, 30^{\circ}, 35^{\circ}\right)$. As for tilting direction, significant effects of azimuth size were found in all the four directions $(\mathrm{p}<.0001)$. As for tilting range, when the tilting range is under $35^{\circ}\left(15^{\circ}, 20^{\circ}, 25^{\circ}, 30^{\circ}\right)$, there were significant effects of azimuth size $(\mathrm{p}<.05)$; however, there was no significant effect of azimuth size in tilting range $35^{\circ}(\mathrm{p}=.315)$.

\section{Co-variation between Tilting Actions and Unintentional Pen Tip Movement}

We also analyzed the relationship between tilting actions and unintentional pen tip movement. This analysis is also necessary because that unintentional pen tip movements could be created in pen rolling actions ( $\mathrm{Bi}$ et al., 2008), which may influence the ongoing interaction task. We suspect the similar actions like pen tilting may also create unintentional pen tip movements. In order to investigate the co-variation between tilting actions and unintentional pen tip movement, we perform following analysis.

\section{Main Effects of Independence Variables}

The mean pen tip movements of four directions (E', S', W', N') are $2.40 \mathrm{~mm}, 3.22 \mathrm{~mm}, 2.80 \mathrm{~mm}$, and $3.41 \mathrm{~mm}$ (Figure 18). Repeated measures analysis of variance showed a significant main effect for directions in pen tip movement (F3, $33=11.85$, p < .0001). Pairwise comparisons indicate that the pen tip movement of each direction is significantly different from others $(p<.05)$, except between $S^{\prime}$ and $N^{\prime}(p=.354)$

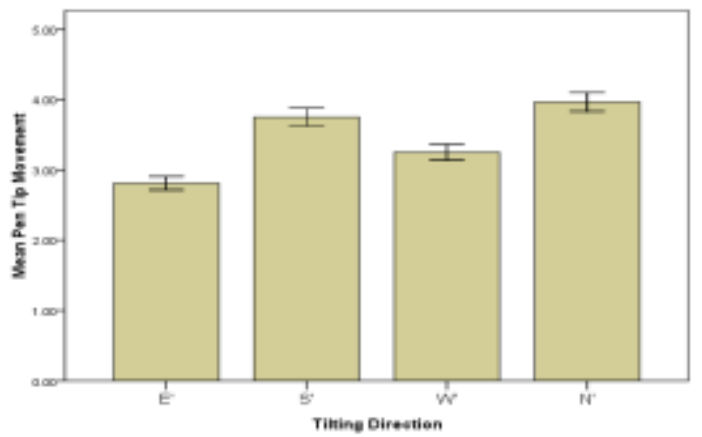

Figure 18. Mean unintentional pen tip movement in four directions.

The mean pen tip movements of five tilting ranges $\left(15^{\circ}, 20^{\circ}, 25^{\circ}\right.$, $30^{\circ}, 35^{\circ}$ ) are $2.58 \mathrm{~mm}, 2.74 \mathrm{~mm}, 2.98 \mathrm{~mm}, 3.12 \mathrm{~mm}$, and $3.38 \mathrm{~mm}$ respectively, as shown in Figure 19. Repeated measures analysis of variance showed a significant main effect for tilting ranges in pen tip movement (F3, $33=24.93, \mathrm{p}<.0001)$. Pairwise comparisons showed that the pen tip movement of each shorter tilting range is significantly shorter than that of others, except of the pen tip movements between $15^{\circ}$ and $20^{\circ}(\mathrm{p}=.107)$.

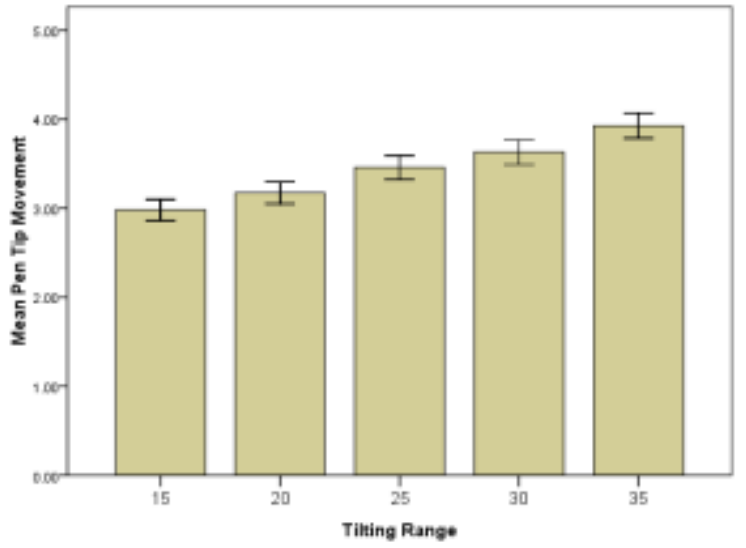

Figure 19. Mean unintentional pen tip movement in five tilting ranges

The mean pen tip movements of four azimuth sizes $\left(30^{\circ}, 45^{\circ}, 60^{\circ}\right.$, $90^{\circ}$ ) are $2.87 \mathrm{~mm}, 2.95 \mathrm{~mm}, 2.98 \mathrm{~mm}$, and $3.04 \mathrm{~mm}$ respectively, as shown in Figure 20. We also explored the influences of azimuth size on pen tip movement. Repeated measures analysis of variance showed no significant main effect for azimuth size in pen tip movement $(\mathrm{F} 3,33=2.05, \mathrm{p}=.126)$. Pairwise comparisons showed no significant different between them.

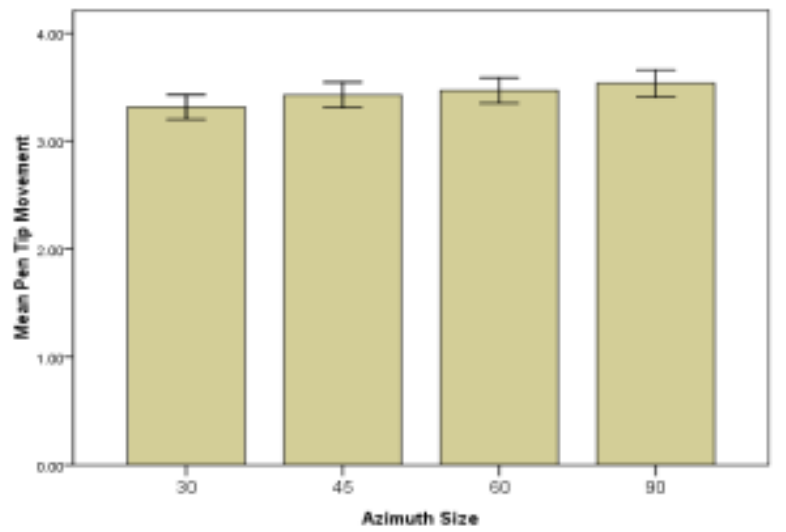

Figure 20. Mean unintentional pen tip movement in four azimuth sizes.

\section{Interaction Effects between Independence Variables}

As for the pen tip movements, repeated measures analysis of variance showed no interaction between tilting range and target azimuth size $(\mathrm{p}=.097)$ (Figure 21$)$, tilting range and direction ( $\mathrm{p}$ $=.212$ ) (Figure 22), and between target azimuth size and direction $(\mathrm{p}=.478)$ (Figure 23). 


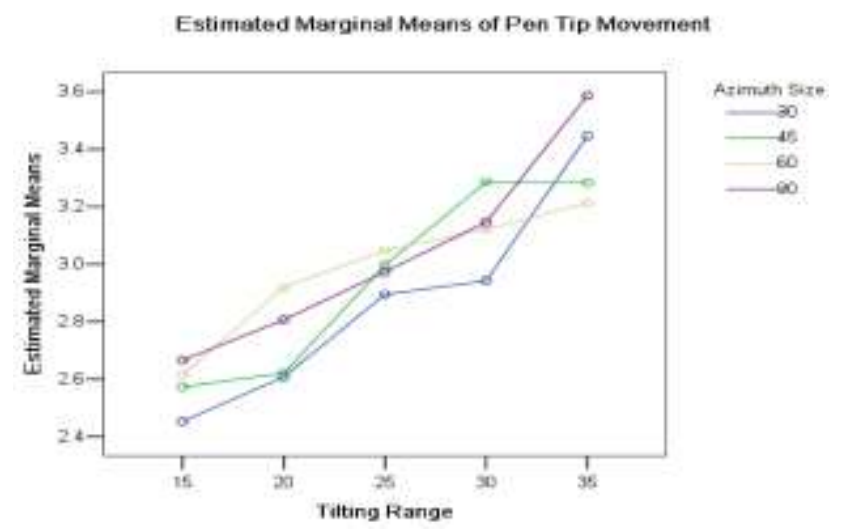

Figure 21. Interaction effects between tilting ranges and target azimuth sizes in pen tip movement.

Estimated Marginal Means of Pen Tip Movement

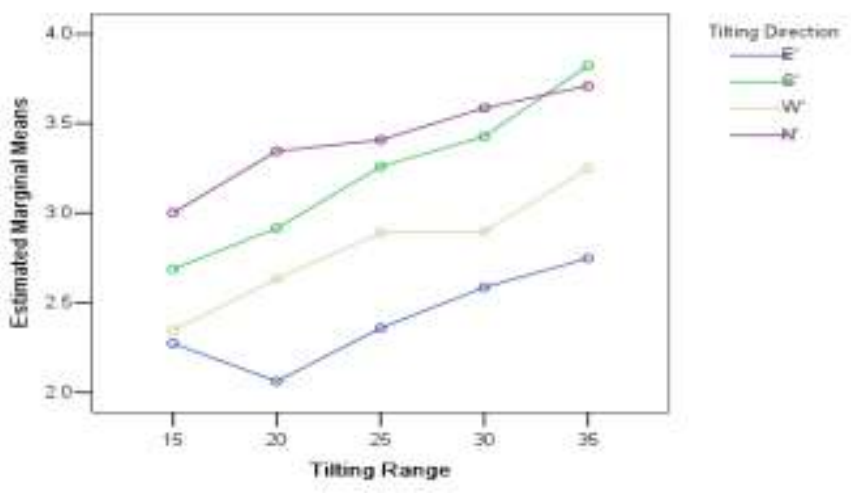

Figure 22. Interaction effects between tilting ranges and directions in pen tip movement.

Estimated Marginal Means of Pen Tip Movement

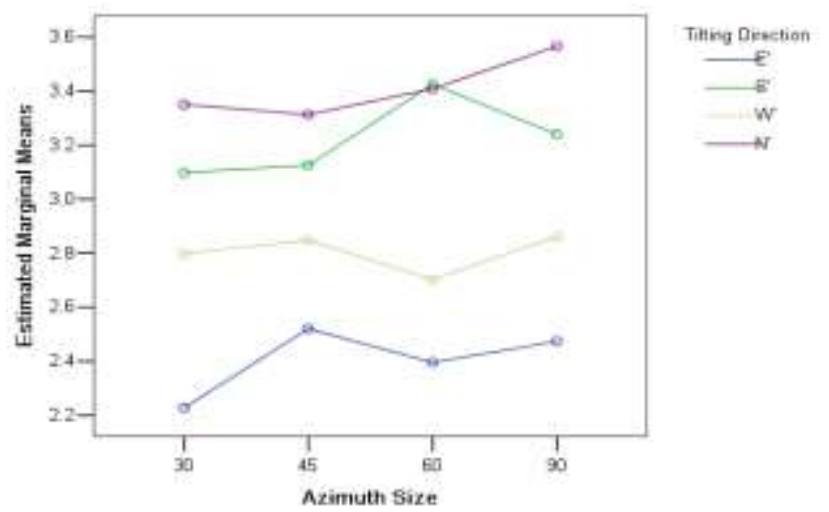

Figure 23. Interaction effects between target azimuth sizes and directions in pen tip movement.

\section{DISCUSSION}

\section{Factors that Affect Task Completion Time}

Results of our studies showed that longer tilting range lead to longer task completion time. Thus, the efficiency of tilting tasks can be improved by shortening tilting ranges. This relationship between task completion time and the distance of target in goal tilting tasks is similar to that in goal reaching tasks governed by the Fitt's law [6] and that in goal following tasks governed by the steering law [1]. However, if the tilting range is small than $20^{\circ}$, incidental tilting actions will be contaminated by intentional tilting actions, which cause high error rates (Figure 13).

Our results also showed that increasing the azimuth size can shorten the task completion time of goal tilting. This finding suggests that in tilt tasks, completion time could be shorted by design larger azimuth sizes. Comparing with the influences on the marking menu [10], and Zone and Polygon menus [23], the accepted azimuth size is far larger. Zhao's work [23] suggests that Zone and Polygon menus can be extended in breadth to 16 items, while providing good speed and accuracy. As we got in the experiment (as shown in Figure 14), when the azimuth size is small like $30^{\circ}$, selection becomes error-prone. It may be partly due to the muscle groups used in tilting tasks. When users perform tilting tasks, the wrist may mainly used to do tilting in different directions, and fingers seem less involved in order to keep the pen tip stable. Zhai et al. [22] presented that relative performance of 6DOF devices were depend on the muscle groups used. Using fingers, wrist and arm tend to outperform using only wrist and arm. Balakrishnan et al. [3] also found that a combined use of multiple fingers resulted in higher performance than other limb segments.

\section{Factors that Affect Task Error Rates}

Our results showed that when azimuth size is $30^{\circ}$, the mean error rate is significant higher than that of others, which is similar to the result of our pervious study of Tilt Menu. Furthermore, our results also showed that only when azimuth size is $30^{\circ}$, there are significant main effect of tilting range $(\mathrm{p}<.0001)$, in contrast, when the azimuth size is above $30^{\circ}\left(45^{\circ}, 60^{\circ}, 90^{\circ}\right)$, it showed no significant main effects of tilting range $(\mathrm{p}=.059, \mathrm{p}=.681, \mathrm{p}=$ .389 ). It's also similar to the results of previous literatures. As mentioned by MacKenzie, an error-rate analysis may also reveal the inequitable contributions of A and W [11], Wade, Newell, and Wallace [20] and Card et al. [5] observed a similar significant main effect between error rate and target width, with errors increasing as target width decreased but no main effect between error rate and target amplitude.

As for the tilting range, results of our studies showed that if the tilting range is $15^{\circ}$, the error rate is significantly higher than that of others. It may partly due to that in such cases, incidental tilting actions will be contaminated by intentional tilting actions.

\section{Preferred Tilting Directions and Directions that should be avoided}

Our results also indicate different tilting performance in different directions. Tilting in the direction S' will lead to significantly longer task completion time than tilting in other directions (Figure 6). Also, by comparing pen tip movements in four directions during tilting, we found that movement in $S^{\prime}$ are significantly larger than those in E' and W'(Figure 18). Thus, tilting performance in $\mathrm{S}^{\prime}$ could be considered as the worst.

Tilting in both E' and W' directions tends to lead better performance than in N'. Tilting in E' consistently outperforms tilting in $\mathrm{N}^{\prime}$ with shorter task completion time (Figure 6), and lower error rates (Figure 12).

Furthermore, E' seems the best direction for tilting. Among various measures we conducted, tilting in E' outperforms in $\mathrm{W}^{\prime}$ in almost every aspects: less task completion time (Figure 6), lower error rates (Figure 12), and shorter pen tip movement (Figure 18). 
When we further looked at the effect of separated azimuth sizes, we found that with the increasing of azimuth sizes, the differences between four directions are decreased. When the azimuth size is equal to $90^{\circ}$, there are no significant differences among four directions. This result aligns with the result in our previous study in which the Tilt Menu has four slices. One explanation to this result is that the obscuration by the right hand becomes less obvious with the increasing of the azimuth size, so when the azimuth size reach to $90^{\circ}$, the factor tends not to influence tilting performance.

Our results showed co-variations existing between tilting actions and unintentional pen tip movement. There are significant differences between four directions in unintentional pen tip movement. If pen tip movement is considered in design, the priority for choosing directions should be E', W', N' and S'. Meanwhile, increasing tilting range can significantly increase unintentional pen tip movement, partly because long tilting distance requires more efforts to control the pen tail and causes more unintentional pen tip movement. However, increasing the azimuth size tends not to significantly influence unintentional pen tip movement.

\section{CONCLUSION}

In this paper, we reported our experimental studies to investigate pen tilting performances in pen-based user interfaces. The baseline study helped to determine tilting directions, tilting ranges, and the thresholds that separate incidental pen tilting actions from intentional actions used for interaction. Based on these important parameters, we conducted an experiment to study how factors like tilting range, target azimuth size, and tilting direction affect task performance.

This research has some limitations. In the future, we will extend our research on user tilting behaviors in the following directions. First, we will study the tilting tasks by left-handed users. Our experiments focused on right-handed users. It would be interesting to explore whether the results from this paper can be extended to left-hand users. Second, we will study user behaviors in touchsensitive devices (e.g., tablet PCs). Our experiments used touch pad, in which pen control and visual display are visually separated in two different surfaces. The mismatches between control and display may affect user performance. When touch-based devices become more and more popular, it will be valuable to understand the characteristics of pen tilting in such devices. Furthermore, tilting tasks in our study were simple. We are interested in studying how pen-tail tilting can be used in more complex situations, such as pen-tip is involved in drawing.

\section{REFERENCES}

[1] Accot, J., Zhai, S., 1997. Beyond Fitts' law: models for trajectory-based HCI tasks. Proceedings of the CHI 1997: ACM Conference on Human Factors in Computing Systems. ACM, New York, pp. 295-302.

[2] Bi, X., Moscovich, T., Ramos, G., Balakrishnan, R., Hinckley, K., 2008. An Exploration of Pen Rolling for Penbased Interaction. In Proc. UIST 2008. 191-200.

[3] Balakrishnan, R., Baudel, T., Kurtenbach, G., Fitzmaurice, G., 1997. The Rockin'Mouse: integral 3D manipulation on a plane. In Proc. CHI 1997, ACM Press, 311-318.

[4] Balakrishnan, R., MacKenzie, I. S., 1997. Performance differences in the fingers, wrist, and forearm in computer input control. In Proceedings of ACM CHI'97 Conference on Human Factors in Computing Systems, pages 303-310.

[5] Card, S. K., English, W. K., \& Burr, B. J. 1978. Evaluation of mouse, rate-controlled isometric joystick, step keys, and text keys for text selection on a CRT. Ergonomics, 21, 601613.

[6] FITTS, P. M., 1954. The information capacity of the human motor system in controlling the amplitude of movement. J. Experiment. Psych. 47, 6 (June), 381-391. (Reprinted in Journal of Experimental Psychology: General, 121(3):262269, 1992).

[7] Gan, K-C., Hoffmann, E.R., 1988. Geometrical conditions for ballistic and visually controlled movements. Ergonomics, 31, 829-839.

[8] Grossman, T., Hinckley, K., Baudisch, P., Agrawala, M., Balakrishnan, R., 2006. Hover widgets: Using the tracking state to extend the capabilities of pen-operated devices. In Proc. CHI 2006, ACM Press, Montréal, pp. 861-860.

[9] Kurtenbach, G., Buxton, W., 1992. The limits of expert performance using hierarchical marking menus. In Proc. CHI 1992, ACM Press(1992), 482-487.

[10] Kurtenbach, G., Sellen, A., Buxton, W., 1993. An empirical evaluation of some articulatory and cognitive aspects of "marking menus". Human Computer Interaction, 1993. 8(1): 1--23.

[11] MACKENZIE, I. S., 1992. Fitts' law as a research and design tool in human-computer interaction. Hum.-Comput. Interact. 7, 91-139.

[12] MEYER, D. E., SMITH, J. E. K., KORNBLUM, S., ABRAMS, R. A., WRIGHT, C. E., 1990. Speed accuracy tradeoffs in aimed movements: Toward a theory of rapid voluntary action. In Attention and Performance XIII, M. Jeannerod, ED. Lawrence Erlbaum, Hillsdale, NJ. 173-226.

[13] Partridge, K., Chatterjee, S., Want, R., 2001. TiltType: Accelerometer-supported text entry for very small devices, In Proc. CHI 2001, ACM Press, 201-204.

[14] Ramos, G., Boulos, M., Balakrishnan, R., 2004. Pressure Widgets. In Proc. CHI 2004, ACM Press, 487-494.

[15] Rahman, Mahfuz, Gustafson, Sean, Irani, Pourang, Subramanian, Sriram, 2009. Tilt techniques: investigating the dexterity of wrist-based input. In Proceedings of ACM CHI 2009 Conference on Human Factors in Computing Systems 2009. pp. 1943-1952.

[16] Rekimoto, J., 1996. Tilting operations for small screen interfaces. Proc. UIST '96, 167-168.

[17] Suzuki, Y., Misue, K., Tanaka, J., 2007. Stylus Enhancement to Enrich Interaction with Computers. In Proc. HCII 2007, 133-142

[18] Tian, F., Ao, X., Wang, H., Setlur, V., Dai G., 2007. The Tilt Cursor: Enhancing Stimulus-Response Compatibility Based on 3D Orientation Cue of Pen Devices. In Proc. CHI 2007. ACM Press (2007), 303-306.

[19] Tian, F., Xu, L., Wang, H., Zhang, X., Liu, Y., Setlur, V., Dai, G., 2008. Tilt Menu: Using the 3D Orientation Information of Pen Devices to Extend the Selection Capability of Pen-based User Interfaces. In Proc. CHI 2008, 1077--1086. 
[20] Wade, M. G., Newell, K. M., \& Wallace, S. A. 1978. Decision time and movement time as a function of response complexity in retarded persons. American Journal of Mental Deficiency, 83, 135-144.

[21] Wigdor, D., Balakrishnan, R., 2003. TiltText: Using Tilt for Text Input to Mobile Phones. In Proc. UIST 2003. ACM Press, 81-90.

[22] Zhai S., Milgram, P., and Buxton, W., 1996. The influence of muscle groups on performance of multiple degree-offreedom input. In Proceedings of ACMCHI'96 Conference on Human Factors in Computing Systems, pages 308-315.

[23] Zhao, S., Agrawala, M., Hinckley, K., 2006. Zone and Polygon Menus: Using Relative Position to Increase the Breadth of Multi-Stroke Marking Menus. In Proc. CHI 2006, ACM Press (2006), 1077-1086.

[24] Zhou X., Ren, X., Hui, Y., 2008. An Empirical Comparison of Pen Pressure and Pen Tilt Input Techniques. ISPA 2008: 982-989. 